\title{
The Topology of the Set of Conditioned Invariant Subspaces
}

\author{
X. Puerta ${ }^{a, 1}$ U. Helmke $^{\mathrm{b}, 2}$ \\ ${ }^{a}$ E.U.P.B. \\ Universitat Politècnica de Catalunya \\ Av. Gregorio Marañon 08028 Barcelona \\ coll@ma1.upc.es \\ ${ }^{\mathrm{b}}$ Mathematisches Institut \\ Universität Würzburg \\ Am Hubland 97074 Würzburg \\ helmke@mathematik.uni-wuerzburg.de
}

\begin{abstract}
We consider the topology of the set of conditioned invariant subspaces of an observable pair $(C, A)$ of a fixed dimension. By fixing the observability indices of the restricted system, a stratification by finitely many smooth manifolds is obtained, termed Brunovsky strata. It is shown that each Brunovsky stratum is homotopy equivalent to a generalized flag manifold. From this description an effective formula for the Betti numbers of the Brunovsky strata can be derived.
\end{abstract}

\section{Introduction}

Conditioned invariant subspaces play an important role in geometric control theory and especially observer theory. They generalize the class of invariant subspaces of a linear operator and are thus of considerable interest both in linear systems theory as well as in linear algebra. The study of conditioned or controlled invariant subspaces has been initiated by Wonham [9] and Basile and Marro and has subsequently led to extensive research in systems theory, including the important extension of such concepts to nonlinear control theory. Despite their obvious importance for the structure and design theory of

1 Research partially supported by the European Nonlinear Control Network

2 Research partially supported by a grant from the German Israeli Foundation GIF I-526-034.06/97

Preprint submitted to Elsevier Preprint 6 March 2000 
linear systems, there are only very few studies on parametrization issues of conditioned invariant subspaces. This is in contrast to the situation of invariant subspaces of a finite dimensional linear operator whose topology has been extensively studied by e.g. Shayman [8]. He proved that the set of invariant subspaces admits a natural decomposition into finitely many smooth manifolds, obtained by fixing the Jordan structure of the restricted operator. In Helmke and Shayman [7] deeper topological properties of these Jordan strata of invariant subspaces have been established by showing that they are homotopy equivalent to a generalized flag manifold.

The first basic results on the parametrization of conditioned invariant subspaces are due to Hinrichsen, Münzner and Prätzel-Wolters [6]. In their paper a module theoretic approach to the parametrization of conditioned invariant subspaces is developed that leads to a finite decomposition into cells. Although the geometric aspects of this parametrization have not been worked out in detail, a study of the topological properties of conditioned invariant subspaces has been carried out by H.F.Münzner in the early 80's. In fact, Münzner constructed a cell decomposition of the set of conditioned invariant subspaces and studied its topological properties, including a combinatorial characterization of the boundary cells of a given cell. However his results did never appear in printed form and were outlined only via one or two lectures given at the universities of Bremen and Beer Sheva. For about a decade there has been no subsequent work on the subject. In the past few years there has been new interest into parametrizing conditioned invariant subspaces. A homeomorphism between observable pairs and conditioned invariant subspaces has been constructed in Fuhrmann and Helmke [1] while Ferrer, Puerta and Puerta [4], [5] have shown that the set of conditioned invariant subspaces with fixed observability indices of the restricted system forms a smooth manifold. As these manifolds are obtained by fixing the restricted dual Brunosvky indices of the system we term them Brunosvky strata.

In this paper we show, by an extension of the work of Helmke and Shayman [7] that there exists a strong deformation retraction of the Brunovsky strata of conditioned invariant subspaces onto a generalized flag manifold. In particular, we prove that Brunovsky strata are homotopy equivalent to generalized flag manifolds. Since the Betti numbers of generalized flag manifolds are known (see Helmke and Shayman [7] ) this enables us also to compute the Betti numbers of Brunovsky strata. Our approach to computing the Betti numbers avoids a more tedious, but also more explicit approach via the construction of a suitable cell decomposition. Moreover, further insight is gained by showing that the Brunovsky strata are homotopy equivalent to a set consisting of subspaces that are simultaneously $A$-invariant and $(C, A)$-invariant. 


\section{Preliminaries}

In this section we recall some basic topological results on sets of $A$-invariant subspaces and conditioned invariant subspaces.

Let $G_{k}\left(F^{n}\right)$ denote the Grassmann manifold of $k$-dimensional linear subspaces of $F^{n}$ ( $F$ denotes the field of real or complex numbers). Let $A \in F^{n \times n}$ and $\bar{A} \in F^{k \times k}$ be nilpotent Jordan matrices with Jordan indices (the sizes of the nilpotent Jordan blocks) $\mu=\left(\mu_{1}, \ldots, \mu_{p}\right), \mu_{1} \geq \ldots \geq \mu_{p}>0$ and $\lambda=$ $\left(\lambda_{1}, \ldots, \lambda_{p}\right), \lambda_{1} \geq \ldots \geq \lambda_{p} \geq 0$ respectively. We assume that each Jordan block in $A$ or $\bar{A}$ is of the lower triangular form

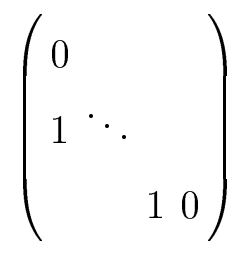

Let $\operatorname{Inv}_{\lambda}(A)$ denote the set of all $k$-dimensional subspaces $V \subset F^{n}$ such that the restriction $\left.A\right|_{V}$ has Jordan indices $\lambda$. It is easily shown that the set is nonempty if and only if $\lambda$ is compatible with $\mu$, i.e. if and only if $\lambda_{i} \leq \mu_{i}$ for $i=1, \ldots, p$.

We first recall the description of $\operatorname{Inv}_{\lambda}(A)$ as an orbit space; see [8]. Let $V_{k}\left(F^{n}\right)$ denote the Stiefel manifold of $n \times k$ full rank matrices $Z$ with entries in $F$. Let

$$
N(\lambda, \mu):=\left\{Z \in V_{k}\left(F^{n}\right) \quad \mid \quad A Z=Z \bar{A}\right\}
$$

and

$$
H(\lambda):=\left\{S \in G l_{k}(F) \quad \mid \quad \bar{A} S=S \bar{A}\right\}
$$

denote the stabilizer subgroup of $\bar{A}$ in $G l_{k}(F) . N(\lambda, \mu)$ and $H(\lambda)$ consist of blocks of lower triangular Toeplitz matrices. The Lie group $H(\lambda)$ acts freely on $N(\lambda, \mu)$ from the right, yielding a smooth quotient manifold

$$
N(\lambda, \mu) / H(\lambda)
$$

Moreover, the inclusion

$$
i: N(\lambda, \mu) \longrightarrow V_{k}\left(F^{n}\right)
$$

induces an embedding of $N(\lambda, \mu) / H(\lambda)$ into the Grassmann manifold $G_{k}\left(F^{n}\right)$ with image $\operatorname{Inv}_{\lambda}(A)$. Thus, $\operatorname{Inv}_{\lambda}(A)$ is a smooth manifold of $G_{k}\left(F^{n}\right)$ and the map $Z \longmapsto I m Z$ induces a diffeomorphism

$$
N(\lambda, \mu) / H(\lambda) \cong \operatorname{Inv}_{\lambda}(A)
$$


In the sequel we will identify $\operatorname{Inv}_{\lambda}(A)$ with its equivalent homogeneous space description as $N(\lambda, \mu) / H(\lambda)$.

We now describe the retraction of $\operatorname{Inv}_{\lambda}(A)$ onto a generalized flag manifold, following [7].

Let $a=\left(a_{1}, \ldots, a_{d}\right), b=\left(b_{1}, \ldots, b_{d}\right)$ be two sequences of integers with $a_{1} \leq$ $\ldots \leq a_{d}, b_{1} \leq \ldots \leq b_{d}$ and $a_{1} \leq b_{1}, \ldots, a_{d} \leq b_{d}=n$. We define

$$
F \operatorname{Flag}(a, b):=\left\{\left(V_{1}, \ldots, V_{d}\right) \in \prod_{i=1}^{d} G_{a_{i}}\left(F^{b_{i}}\right) \quad \mid \quad V_{1} \subset \ldots \subset V_{d}\right\} .
$$

Flag $(a, b)$ is called a generalized flag manifold and is indeed a compact manifold of dimension $\left(a_{0}:=0\right)$

$$
\sum_{i=1}^{d}\left(a_{i}-a_{i-1}\right)\left(b_{i}-a_{i}\right)
$$

For explicit formulas of the Betti numbers of Flag $(a, b)$ we refer to [7]. Flag $(a, b)$ has the following description as an orbit space. Let $V(a, b)$ consist of those matrices $X \in V_{a_{d}}\left(F^{n}\right)$ such that the last $n-b_{i}$ entries in columns $a_{i-1}+1, \ldots, a_{i}$ are equal to $0, i=1, \ldots, d$. For $b=a, P(a):=V(a, a)$ is a parabolic subgroup of $G l_{a_{d}}(F)$ and $F \operatorname{lag}(a, b)$ is diffeomorphic to the orbit space $V(a, b) / P(a)$.

Theorem 1 [7] Let $\left(\mu_{1}^{*}, \ldots, \mu_{p^{*}}^{*}\right)$ and $\left(\lambda_{1}^{*}, \ldots, \lambda_{p^{*}}^{*}\right)$ denote the dual partitions of $\mu$ and $\lambda$ respectively. Let $a_{i}=\lambda_{p^{*}-i+1}^{*}$ and $b_{i}=\mu_{p^{*}-i+1}^{*}, 1 \leq i \leq p^{*}$, then Flag $(a, b)$ is a strong deformation retract of $\operatorname{Inv}_{\lambda}(A)$.

We briefly summarize the results on the orbit structure of conditioned invariant subspaces, as described in [4].

Let $(C, A) \in F^{p \times n} \times F^{n \times n}$ be an observable pair of matrices in dual Brunovsky canonical form. Let $\mu=\left(\mu_{1}, \ldots, \mu_{p}\right), \mu_{1} \geq \ldots \geq \mu_{p}>0$ denote the observability indices of $(C, A)$. Notice that the $\mu_{i}$ coincide with the Jordan indices of $A$. Recall that a subspace $V \subset F^{n}$ is $(C, A)$-invariant if there exists an output injection matrix $J$ such that $V$ is $(A+J C)$-invariant. The restriction of $(C, A)$ to $V$ is the pair $\left.(C, A)\right|_{V}:=\left(\left.C\right|_{V},\left.(A+J C)\right|_{V}\right)$. Although is not uniquely determined some basic properties of the restricted system are invariant under output injection. If $(C, A)$ is observable then the restriction $\left.(C, A)\right|_{V}$ is observable. Moreover the observability indices $\lambda_{1} \geq \ldots \geq \lambda_{p} \geq 0$ of the restricted subsystem are well defined and independent of the choice of $J$. We refer to $\lambda$ as the restricted indices of $(C, A)$ on $V$. We note without proof that the restricted indices $\lambda_{1}, \ldots, \lambda_{p}$ of a $(C, A)$-invariant subspaces satisfy the inequalities $\lambda_{i} \leq \mu_{i}$ for $i=1, \ldots, p$. Moreover for any $\lambda=\left(\lambda_{1}, \ldots, \lambda_{p}\right)$ with $\lambda_{1} \geq \ldots \geq \lambda_{p} \geq 0$ and $\lambda_{i} \leq \mu_{i}$ there exists a $(C, A)$-invariant subspace $V$ so that $\lambda_{i}$ are the restricted observability indices. 
Let

and

$$
\operatorname{Inv}_{k}(C, A):=\left\{V \in G_{k}\left(F^{n}\right) \quad \mid \quad V \text { is }(C, A) \text {-invariant }\right\}
$$

$$
\operatorname{Inv}_{\lambda}(C, A):=\left\{V \in \operatorname{Inv}_{k}(C, A) \quad|\quad(C, A)|_{V} \text { has restricted indices } \lambda\right\} .
$$

$\operatorname{Inv}_{\lambda}(C, A)$ defines a finite partition of $\operatorname{Inv}_{k}(C, A)$ into disjoint subsets

$$
\operatorname{Inv}_{k}(C, A)=\bigcup_{\lambda \leq \mu} \operatorname{Inv}_{\lambda}(C, A)
$$

The space $\operatorname{Inv}_{\lambda}(C, A)$ has a description as an orbit space similar to that of $\operatorname{Inv}_{\lambda}(A)$; see [4],[5]. Let $(\bar{C}, \bar{A})$ denote the Brunovsky canonical form of $\left.(C, A)\right|_{V}, V \in \operatorname{Inv}_{\lambda}(C, A)$.

Let $M(\lambda, \mu)$ be the set of matrices $Z \in F^{n \times k}$ with

$$
\text { (1) }\left\{\begin{array}{l}
A Z=Z \bar{A}+A Z \bar{C}^{t} \bar{C} \\
C Z=C Z \bar{C}^{t} \bar{C}
\end{array}\right.
$$

and such that $C Z \bar{C}^{t}$ has full rank. Let $G(\lambda):=M(\lambda, \lambda)$ denote the set of matrices $S \in G l_{k}(F)$ with

$$
\left\{\begin{array}{l}
\bar{A} S=S \bar{A}+\bar{A} S \bar{C}^{t} \bar{C} \\
\bar{C} S=\bar{C} S \bar{C}^{t} \bar{C}
\end{array}\right.
$$

$M(\lambda, \mu)$ and $G(\lambda)$ consists of blocks of upper triangular Toeplitz matrices. $G(\lambda)$ forms a Lie subgroup of $G l_{k}(F)$ that acts freely on $M(\lambda, \mu)$ from the right. It is in fact the largest subgroup of $G l_{k}(F)$ that acts on $M(\lambda, \mu)$ via $Z \longmapsto Z S^{-1}$. Thus, if $S \in G l_{k}(F)$ is a transformation such that for $Z \in$ $M(\lambda, \mu), Z S \in M(\lambda, \mu)$, then $S \in G(\lambda)$. The orbit space of the above group action defines a smooth quotient manifold

$$
M(\lambda, \mu) / G(\lambda)
$$

and the inclusion

$$
i: M(\lambda, \mu) \longrightarrow V_{k}\left(F^{n}\right)
$$

induces an embedding of $M(\lambda, \mu) / G(\lambda)$ into the Grassmann manifold $G_{k}\left(F^{n}\right)$ with image $\operatorname{Inv}_{\lambda}(C, A)$. Thus, $\operatorname{Inv}_{\lambda}(C, A)$ is a smooth submanifold of $G_{k}\left(F^{n}\right)$ and the map $Z \longmapsto I m Z$ defines a diffeomorphism

$$
M(\lambda, \mu) / G(\lambda) \cong \operatorname{Inv}_{\lambda}(C, A)
$$

In the sequel we will identify $\operatorname{Inv}_{\lambda}(C, A)$ with its equivalent homogeneous space description as $M(\lambda, \mu) / G(\lambda)$. 


\section{Main result}

Having introduced the Brunovsky strata as sets of conditioned invariant subspaces with fixed restriction indices, we prove that there is a natural retraction of Brunovsky strata onto a generalized flag manifold. Let $\left(\mu_{1}^{*}, \ldots, \mu_{p^{*}}^{*}\right)$ and $\left(\lambda_{1}^{*}, \ldots, \lambda_{p^{*}}^{*}\right)$ denote the dual partitions of $\mu$ and $\lambda$ respectively. Our main result can be stated as follows.

Theorem 2 Flag $(a, b)$ is a strong deformation retract of $\operatorname{Inv}_{\lambda}(A, C)$, where $a_{i}=\lambda_{p^{*}-i+1}^{*}$, and $b_{i}=\mu_{p^{*}-i+1}^{*}, 1 \leq i \leq p^{*}$. In particular, $\operatorname{Inv}_{\lambda}(A, C)$ is homotopy equivalent to Flag $(a, b)$ and these spaces have the same Betti numbers.

The proof of the theorem depends on following lemmas.

The first lemma is essentially a reformulation of a result from [4]. We therefore do not give a proof here.

Recall, that $V(a, b)$ denotes the set of all full rank $n \times a_{d}$ matrices, such that the last $n-b_{i}$ entries in columns $a_{i-1}+1, \ldots, a_{i}$ are equal to $0, i=1, \ldots, d$.

Lemma 3 Let $Z \in M(\lambda, \mu)$, then $C Z \bar{C}^{t} \in V(a, b)$ where $a_{i}=\lambda_{p^{*}-i+1}^{*}$, and $b_{i}=\mu_{p^{*}-i+1}^{*}, 1 \leq i \leq p^{*}$. Analogously if $Z \in G(\lambda)$ then $C Z \bar{C}^{t} \in P(a)$.

Consider the surjective smooth maps

$$
\begin{gathered}
\gamma: M(\lambda, \mu) \longrightarrow V(a, b) \\
\gamma: G(\lambda, \mu) \longrightarrow P(a)
\end{gathered}
$$

defined by $\gamma(Z)=\bar{C} Z \bar{C}^{t}$.

Lemma 4 Let $Z \in M(\lambda, \mu)$ and $T \in G(\lambda)$, then $\gamma(Z T)=\gamma(Z) \gamma(T)$.

Proof Multiplying the equation $C Z=C Z \bar{C}^{t} \bar{C}$ of (1) by $T \bar{C}^{t}$ on the right we have that $C Z T \bar{C}^{t}=C Z \bar{C}^{t} C T \bar{C}^{t}$; this is to say, $\gamma(Z T)=\gamma(Z) \gamma(T)$.

As a consequence of the previous lemma we obtain an induced map on quotients

$$
\tilde{\gamma}: \operatorname{Inv}_{\lambda}(A, C) \longrightarrow V(a, b) / P(a) \cong F \operatorname{Fag}(a, b)
$$

defined by $\tilde{\gamma}\left([Z]_{G(\lambda)}\right)=[\gamma(Z)]_{P(a)}$. We claim that $\tilde{\gamma}$ define a strong deformation retract. This then implys our main result. To show this we first identify $V(a, b) / P(a)$ with a submanifold of $\operatorname{Inv}_{\lambda}(A, C)$. In fact, we will show that this submanifold is just the intersection $\operatorname{Inv}_{\lambda}(A, C) \cap \operatorname{Inv}_{\lambda}(A)$. 
We are interested in studying the structure of the intersection $M(\lambda, \mu) \cap$ $N(\lambda, \mu)$. Note that since $(\bar{C}, \bar{A})$ is in Brunovsky canonical form we have that $\bar{A} \bar{C}^{t}=0$. Thus we obtain the description of $M(\lambda, \mu) \cap N(\lambda, \mu)$ as full rank solutions of the system

$$
\left\{\begin{array}{l}
A Z=Z \bar{A} \\
C Z=C Z \bar{C}^{t} \bar{C}
\end{array}\right.
$$

Moreover, since $Z=\left(Z_{i, j}\right)_{i, j} \in N(\lambda, \mu)$ implies that $Z_{i, j}$ is in lower Toeplitz form, while $Z \in M(\lambda, \mu)$ implies that $Z_{i, j}$ is in upper Toeplitz form, the elements $Z \in M(\lambda, \mu) \cap N(\lambda, \mu)$ are blocks of 'diagonal' matrices,

$$
Z_{i, j}=\left(\begin{array}{ccc}
0 & \cdots & 0 \\
\vdots & & \vdots \\
x_{\mu_{i}-\lambda_{j}+1} & & \vdots \\
\vdots & \ddots & \vdots \\
0 & \cdots & x_{\mu_{i}-\lambda_{j}+1}
\end{array}\right) \text { if } \mu_{i} \leq \lambda_{j} \text { or } Z_{i, j}=0 \text { elsewhere. }
$$

Lemma 5 The restriction of $\gamma$ to $M(\lambda, \mu) \cap N(\lambda, \mu)$ is a smooth bijection between $M(\lambda, \mu) \cap N(\lambda, \mu)$ and $V(a, b)$.

Proof Let $Z=\left(Z_{i, j}\right)_{i, j} \in M(\lambda, \mu) \cap N(\lambda, \mu)$. Since the entries of $\gamma(Z)=$ $C Z \bar{C}^{t}$ are the right-bottom corner entries of each block $Z_{i, j}$ of $Z$, we have that $\gamma(Z)$ has the same non zero parameters than $Z$ and therefore the bijectivity of the map holds.

Example 6 if $\mu=(4,3), \lambda=(3,2)$ Then every element of $N(\lambda, \mu) \cap M(\lambda, \mu)$ has the form

$$
\left(\begin{array}{ccc|cc}
0 & 0 & 0 & 0 & 0 \\
x_{1} & 0 & 0 & 0 & 0 \\
0 & x_{1} & 0 & z_{1} & 0 \\
0 & 0 & x_{1} & 0 & z_{1} \\
\hline x_{3} & 0 & 0 & 0 & 0 \\
0 & x_{3} & 0 & z_{2} & 0 \\
0 & 0 & x_{3} & 0 & z_{2}
\end{array}\right)
$$

Since $G(\lambda) \cap H(\lambda)$ acts on $M(\lambda, \mu) \cap N(\lambda, \mu)$ on the right, we obtain:

Corollary 7 induces a bijection between $M(\lambda, \mu) \cap N(\lambda, \mu) / G(\lambda) \cap H(\lambda)$ and $V(a, b) / P(a)$. 
Lemma 8 The inclusion $M(\lambda, \mu) \cap N(\lambda, \mu) \subset M(\lambda, \mu)$ induces an embedding

$$
j:(M(\lambda, \mu) \cap N(\lambda, \mu)) /(G(\lambda) \cap H(\lambda)) \longrightarrow M(\lambda, \mu) / G(\lambda) .
$$

Proof Let us denote $M:=M(\lambda, \mu), N:=N(\lambda, \mu), G:=G(\lambda), H:=H(\lambda)$. We define the map $j:(M \cap N) /(G \cap H) \longrightarrow M / G$ by $j\left([Z]_{G \cap H}\right)=[Z]_{G}$. We remark that the image of $j$ is $(M \cap N) G / G$. Moreover, $j$ is injective since if $Z \in M \cap N$ and $Y \in G l(d), Z Y \in M \cap N$ implies that $Y \in G \cap H$. Let us consider the following commutative diagram.

$$
\begin{array}{ccc}
M \cap N & \hookrightarrow & M \\
p_{1} \downarrow & & p_{2} \downarrow \\
(M \cap N) /(G \cap H) \stackrel{j}{\rightarrow} & M / G
\end{array}
$$

where $p_{1}$ and $p_{2}$ are the projections of the principal bundles defined by the group actions. The smoothness of $j$ follows from the local triviality of $p_{1}$ and $p_{2}$. To prove that $j$ is an embedding, we define local representations of $j$, $\sigma_{2} \circ j \circ \sigma_{1}^{-1}$ where $\sigma_{1}$ and $\sigma_{2}$ are suitable local charts of $(M \cap N) /(G \cap H)$ and $M / G$ respectively. We consider the usual hermitian inner product in $F^{(n+p) \times d}$. Let $Z \in M \cap N$ and $L$ the linear subspace of $\overline{M \cap N}$ orthogonal to $[Z]_{G \cap H}$ at $Z$. Then there exist an open neighborhood $U$ of $Z$ in $L$ such that the map $\sigma_{1}: p_{1}(U) \rightarrow U$ defined intersecting each orbit of $p_{1}(U)$ with $U$ is a local section of $p_{1}$.

Since $j$ is injective, then $L \cap[Z]_{G}=Z$. Let $M$ be the largest linear variety of $\bar{M}$ containing $L$ such that $M \cap[G]_{G}=Z$ (notice that $M$ is an open set of a linear subspace). Then there exist a neighborhood $V$ of $Z$ in $M$ such that the map $\sigma_{2}: p_{2}(V) \rightarrow V$ defined by intersecting each orbit of $p_{2}(V)$ with $V$ is a local section of $p_{2}$.

By shrinking the neighborhoods if necessary, it is clear that $U=V \cap L$ is embedded in $V$ and this embedding is the composition $\sigma_{2} \circ j \circ \sigma_{1}^{-1}$ which represents locally $j$.

After these preparatory results we are now ready to prove our main theorem.

\section{Proof of the main theorem}

From the diffeomorphisms

$$
\operatorname{Inv}_{\lambda}(A, C) \cong M(\lambda, \mu) / G(\lambda)
$$


and

$$
F \operatorname{lag}(a, b) \cong V(a, b) / P(a) \cong(M(\lambda, \mu) \cap N(\lambda, \mu)) /(G(\lambda) \cap H(\lambda))
$$

(Corollary 7)

$$
(M(\lambda, \mu) \cap N(\lambda, \mu)) /(G(\lambda) \cap H(\lambda)) \cong(M(\lambda, \mu) \cap N(\lambda, \mu)) G(\lambda) / G(\lambda)
$$

(Lemma 8), it suffices to construct a deformation retract of $M(\lambda, \mu) / G(\lambda)$ onto $(M(\lambda, \mu) \cap N(\lambda, \mu)) G(\lambda) / G(\lambda)$.

Let us denote by $\sigma$ the inverse of $\left.\gamma\right|_{M(\lambda, \mu) \cap N(\lambda, \mu)}$, which exists by Lemma 5 . Let

$$
H:[0,1] \times M(\lambda, \mu) \longrightarrow M(\lambda, \mu)
$$

be the map defined by $H(t, Z)=D_{\mu}(t) Z D_{\lambda}(t)^{-1}$ where

$$
\begin{aligned}
& D_{\lambda}(t)=\operatorname{diag}\left(D_{\lambda_{1}}(t), \ldots, D_{\lambda_{p}}(t)\right), D_{\lambda_{i}}(t)=\operatorname{diag}\left(t^{\lambda_{i}-1}, t^{\lambda_{i}-2}, \ldots, 1\right) \\
& D_{\mu}(t)=\operatorname{diag}\left(D_{\mu_{1}}(t), \ldots, D_{\mu_{p}}(t)\right), D_{\mu_{i}}(t)=\operatorname{diag}\left(t^{\mu_{i}-1}, t^{\mu_{i}-2}, \ldots, 1\right) .
\end{aligned}
$$

(if $\lambda_{i}=0$ then $D_{\lambda_{i}}(t)$ does not apear in $D_{\lambda}(t)$ ).

We claim that $H$ is smooth with $H(1, Z)=Z, H(0, Z)=\sigma \gamma(Z)$ for all $Z \in M(\lambda, \mu)$ and $H(t, Z) \in M(\lambda, \mu)$ for all $t \in[0,1]$ and $Z \in M(\lambda, \mu)$.

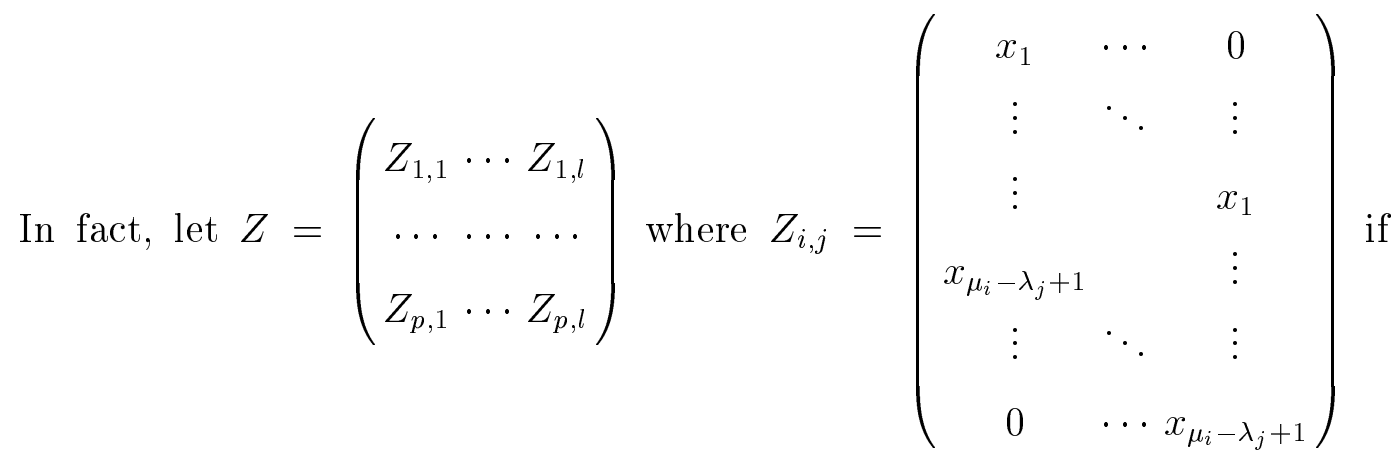
$\mu_{i} \geq \lambda_{j}$ or $Z_{i, j}=0$ elsewhere.

Then $D_{\mu}(t) Z D_{\lambda}(t)^{-1}=\left(D_{\mu_{i}}(t) Z_{i, j} D_{\lambda_{j}}(t)^{-1}\right)_{i, j}$ where

$$
D_{\mu_{i}}(t) Z_{i, j} D_{\lambda_{j}}(t)^{-1}=\left(\begin{array}{ccc}
x_{1} t^{\mu_{i}-\lambda_{j}} & \cdots & 0 \\
\vdots & \ddots & \vdots \\
\vdots & & x_{1} t^{\mu_{i}-\lambda_{j}} \\
x_{\mu_{i}-\lambda_{j}+1} & & \vdots \\
\vdots & \ddots & \vdots \\
0 & \cdots & x_{\mu_{i}-\lambda_{j}+1}
\end{array}\right) .
$$


Since for all the nonzero block $\mu_{i} \geq \lambda_{j}$ it follows that $D_{\mu}(t) Z D_{\lambda}(t)^{-1}$ is a polynomial in $t$, and therefore, $H$ is a smooth map. Moreover, one can easily check that $H(1, Z)=Z$ and $H(0, Z)=\sigma \gamma(Z)$ for all $Z \in M(\lambda, \mu)$ and $H(t, Z) \in M(\lambda, \mu)$ for all $t \in[0,1]$ and $Z \in M(\lambda, \mu)$.

Thus, since for all $T \in G(\lambda)$ and $Z \in M(\lambda, \mu)$

$$
D_{\mu}(t) Z T D_{\lambda}(t)^{-1}=D_{\mu}(t) Z D_{\lambda}(t)^{-1}\left(D_{\lambda}(t) T D_{\lambda}(t)^{-1}\right)
$$

and $D_{\lambda}(t) T D_{\lambda}(t)^{-1} \in G(\lambda)$ we conclude that $H$ induces a well defined map

$$
\tilde{H}:[0,1] \times M(\mu, \lambda) / G(\lambda) \longrightarrow M(\mu, \lambda) / G(\lambda), \quad \tilde{H}\left(t,[Z]_{G(\lambda)}\right)=[H(t, Z)]_{G(\lambda)} .
$$

Finally, we prove the smoothness of $\tilde{H}$. But this follows immediately from the commutative diagram:

$$
\begin{array}{ccc}
{[0,1] \times M(\lambda, \mu)} & \stackrel{H}{\longrightarrow} & {[0,1] \times M(\lambda, \mu)} \\
i d \times \pi \downarrow & i d \times \pi \downarrow \\
{[0,1] \times M(\lambda, \mu) / G(\lambda) \stackrel{\tilde{H}}{\longrightarrow}[0,1] \times M(\lambda, \mu) / G(\lambda)}
\end{array}
$$

where $\pi$ is the projection induced by the group action of $G(\lambda)$ on $M(\lambda, \mu)$. By the local triviality of the principal bundle $\pi$, we conclude that $\tilde{H}$ is smooth .

Example 9 Let $n=7, k=5, \mu=(4,3), \lambda=(3,2)$. Then $\mu^{*}=(2,2,2,1)$, $\lambda^{*}=(2,2,1)$ and $a=(0,1,2,2), b=(1,2,2,2)$. By theorems 1 and 2 both $\operatorname{Inv}_{\lambda}(A)$ and $\operatorname{Inv}_{\lambda}(C, A)$ can be strongly retracted to Flag $(a, b) \cong V(a, b) / P(a)$ where $V(a, b)$ and $P(a)$ are matrices of the form

$$
\left(\begin{array}{cc}
x_{1} & z_{1} \\
x_{2} & z_{2}
\end{array}\right) \text { and }\left(\begin{array}{cc}
x_{1} & z_{1} \\
0 & z_{2}
\end{array}\right) \text { respectively. }
$$

Thus $\operatorname{Inv}_{\lambda}(A)$ and $\operatorname{Inv}_{\lambda}(C, A)$ are homotopy equivalent to the projective line $P^{1}(F)$.

Example $10 \operatorname{Inv}_{\lambda}(A)$ and $\operatorname{Inv}_{\lambda}(C, A)$ are homotopy equivalent to a Grassmannian $G_{r}\left(F^{s}\right)$ if and only if $\lambda^{*}=\left(\lambda_{1}^{*}, \lambda_{2}^{*}, \ldots, \lambda_{l}^{*}, r\right)$ and $\mu^{*}=\left(\lambda_{1}^{*}, \lambda_{2}^{*}, \ldots, \lambda_{l}^{*}, s, \ldots\right)$.

It follows from our main result and [7] that the image of the embedding of $\operatorname{Flag}(a, b)$ in $\operatorname{Inv}_{\lambda}(C, A)$ is a strong deformation retract of $\operatorname{Inv}_{\lambda}(C, A)$ as well as of $\operatorname{Inv}_{\lambda}(A)$. We now prove that this set is in fact the intersection $\operatorname{Inv}_{\lambda}(C, A) \cap \operatorname{Inv}_{\lambda}(A)$.

Theorem 11 Flag $(a, b)$ is diffeomorphic to $\operatorname{Inv}_{\lambda}(C, A) \cap \operatorname{Inv}_{\lambda}(A)$, where a and $b$ are as in theorem 2. 


\section{Proof}

Denote by $j^{\prime}$ the embedding of $V(a, b) / P(a)$ in $M(\lambda, \mu) / G(\lambda)$ defined by the Corollary 7 and the Lemma 8, Denote by $i$ the embedding of $M(\lambda, \mu) / G(\lambda)$ in $G_{k}\left(F^{n}\right)$ defined in [5]. It is sufficient to prove that $i \circ j^{\prime}(V(a, b) / P(a))=$ $\operatorname{Inv}_{\lambda}(C, A) \cap \operatorname{Inv}_{\lambda}(A)$

The inclusion $\subset$ is clear since $V \in i \circ j^{\prime}(V(a, b) / P(a))$ if and only if $V=\operatorname{Im} Z$ where $Z \in M(\lambda, \mu) \cap N(\lambda, \mu)$.

To prove the inclusion $\supset$ we represent every $V \in \operatorname{Inv}_{\lambda}(C, A) \cap \operatorname{Inv}_{\lambda}(A)$ as $V=\operatorname{Im} Z$ where $Z \in M(\lambda, \mu) \cap N(\lambda, \mu)$ satisfies

$$
\left\{\begin{array}{l}
A Z=Z \bar{A} \\
C Z=C Z \bar{C}^{t} \bar{C}
\end{array}\right.
$$

with $C Z \bar{C}^{t}$ having full rank. Let $l:=\operatorname{rk} \bar{C}$. The above relations are equivalent to the simultaneous commutativity of the following diagrams

$$
\begin{aligned}
& F^{k} \stackrel{\bar{A}}{\longrightarrow} F^{k} \quad F^{k} \stackrel{\left(\bar{A}^{t}, \bar{C}^{t}\right)^{t}}{\longrightarrow} F^{(k+l)} \\
& \begin{array}{rrrrr} 
& \downarrow Z & \downarrow Z & \downarrow Z & \downarrow Z^{\prime} \\
& F^{n} \stackrel{A}{\longrightarrow} F^{n} & F^{n} \stackrel{\left(A^{t}, C^{t}\right)^{t}}{\longrightarrow} F^{(n+p)}
\end{array} \quad \text { where } Z^{\prime}=\left(\begin{array}{c}
Z A Z \bar{C}^{t} \\
0 C Z \bar{C}^{t}
\end{array}\right)
\end{aligned}
$$

Therefore we can interpret the columns of $Z$ as a Jordan basis of $\left.A\right|_{V}$ which is simultaneously a Brunovsky basis of $\left.(C, A)\right|_{V}[4]$. We are going to construct such a basis under the assumption that $V \in \operatorname{Inv}_{\lambda}(C, A) \cap \operatorname{Inv}_{\lambda}(A)$.

Let $A_{V}$ and $C_{V}$ denote the linear maps $\left.A\right|_{V}$ and $\left.C\right|_{V}$ respectively. We claim that

$$
F^{n}=\operatorname{Ker} A_{V}^{i} \oplus \operatorname{Ker}\left(\begin{array}{c}
C_{V} \\
C A_{V} \\
\vdots \\
C A_{V}^{i-1}
\end{array}\right)
$$

In fact, since $\left.A\right|_{V}$ and $\left.(C, A)\right|_{V}$ have the same invariants we have that $\operatorname{dim} \operatorname{Ker} A_{V}=$ $n-\operatorname{dim} \operatorname{Ker} C_{V}$ and inductively

$\operatorname{dim} \operatorname{Ker} A_{V}^{i}-\operatorname{dim} \operatorname{Ker} A_{V}^{i-1}=\operatorname{dim} \operatorname{Ker}\left(\begin{array}{c}C_{V} \\ C A_{V} \\ \vdots \\ C A_{V}^{i-2}\end{array}\right)-\operatorname{dim} K e r\left(\begin{array}{c}C_{V} \\ C A_{V} \\ \vdots \\ C A_{V}^{i-1}\end{array}\right) \Rightarrow$ 


$$
\Rightarrow \operatorname{dim} \operatorname{Ker} A_{V}^{i}=n-\operatorname{dim} \operatorname{Ker}\left(\begin{array}{c}
C_{V} \\
C A_{V} \\
\vdots \\
C A_{V}^{i-1}
\end{array}\right)
$$

We can therefore construct following [3], a basis of $V$ formed by chains of vectors of the type $e, A e, A^{2} e, \ldots, A^{i} e \in \operatorname{Ker} C$ with $A^{i+1} e=0$ and $C A^{i+1} e \neq 0$ and a basis of $F^{l}$ consisting on vectors $C A^{i+1} e$. With regard to these bases the matrix of $\left.A\right|_{V}$ is $\bar{A}$ and the matrix representation of $\left.(C, A)\right|_{V}$ is $(\bar{C}, \bar{A})$.

Arranging the vectors of the above basis as the columns of $Z$ we obtain the commutative diagram (3) and therefore $Z \in M(\lambda, \mu) \cap N(\lambda, \mu)$ holds. This completes the proof.

\section{References}

[1] P.A. Fuhrmann and U. Helmke, A homeomorphism between observable pairs and conditioned invariant subspaces, Sys. and Contr. Letts., 30, 217-223 (1997)

[2] P.A. Fuhrmann and U. Helmke, On the parametrization of conditioned invariant subspaces, Preprint.

[3] J. Ferrer and F. Puerta, Similarity of non-everywhere Defined Linear MapsLin.Alg.Appl. (168):27-55, (1992)

[4] J. Ferrer, F. Puerta and X. Puerta, Differentiable structure of the set of controllable $(A, B)^{t}$-invariant subspaces, Lin. Alg. Appl., vol 275-276, 16117r. (1998)

[5] J. Ferrer, F. Puerta and X. Puerta, Stratification of the set of general $(A, B)$ invariant subspaces, preprint.

[6] D. Hinrichsen, H.F. Münzer and D. Prätzel-Wolters, Parametrization of $(C, A)-$ invariant subspaces, Sys. and Contr. Letts., 1, 192-199. (1981)

[7] U. Helmke and M. Shayman, The biflag manifold and the fixed point set of a nilpotent transformation on the flag manifold, Lin. Alg. Appl. 92, 125-159. (1987)

[8] M. Shayman, On the variety of invariant subspaces of a finite-dimensional linear operator, Trans.of AMS (274.2)721-747, (1982)

[9] W.M. Wonham, Linear Multivariable Control Theory: a Geometric Approach, Prentice-Hall, (1974) 